\title{
ORIENTAÇÃO DE IMAGENS PRISM - ALOS UTILIZANDO DIFERENTES MODELOS DE PLATAFORMA
}

\author{
Orientation of PRISM - ALOS images using different platform models
}

\author{
Tiago Lima Rodrigues ${ }^{1}$ \\ Edson Aparecido Mitishita ${ }^{2}$ \\ Luiz Danilo Damasceno Ferreira ${ }^{2}$ \\ 1 Universidade Federal Rural do Rio de Janeiro - UFRRJ \\ Instituto de Tecnologia, Departamento de Engenharia \\ BR 465, km 7, 23890-000, Seropédica, RJ, Brasil \\ 2 Universidade Federal do Paraná \\ Curso de Pós-Graduação em Ciências Geodésicas \\ Caixa Postal 515 - 12201-970 - Curitiba - PR, Brasil
}

\section{Resumo:}

Imagens obtidas por sensores de varredura linear orbitais assumem característica dinâmica, uma vez que cada linha obtida pelo CCD apresenta um conjunto de parâmetros de orientação exterior (POE) diferentes. A modelagem matemática da estimativa dos POE para cada instante durante o tempo de formação da imagem tem sido alvo de pesquisas durante as duas últimas décadas. Este trabalho investigou a modelagem a partir da utilização da teoria do Movimento Uniformemente Variado (MUV), associando as acelerações desta às equações de Dinâmica Orbital. Primeiramente foi considerada uma órbita sem perturbações (Movimento Kepleriano) e posteriormente uma órbita com a perturbação proveniente da atração gravitacional terrestre levando em conta a sua elipticidade, representado pelo harmônico zonal de grau 2 (J2), a qual é indicada como uma das mais significativas perturbações. Também foram testados e comparados modelos de plataforma polinomiais. A partir do uso dos diferentes modelos de plataforma foram realizados diversos experimentos com duas imagens PRISM-ALOS. Com base em uma distribuição de pontos de verificação sobre as imagens, as exatidões planimétricas foram calculadas. De forma quase geral, os valores estimados de erro quadrático médio para os pontos de verificação foram muito próximos em termos de GSD (Ground Sample Distance).

Palavras-chave: Orientação exterior, PRISM-ALOS, Harmônico Zonal J2.

\begin{abstract}
:
Images obtained by orbital pushbroom sensor assume linear dynamic characteristic, since each line obtained by the CCD presents a different set of exterior orientation parameters (EOP). The mathematical modeling of the estimate of EOP for each point during the image formation time has been the subject of research over the last two decades. This work investigated the modeling from the use of the theory of Uniformly Accelerated Motion, involving the acceleration of this theory to the equations of Orbital Dynamics, considering an undisturbed orbit (Keplerian
\end{abstract}


motion) and an orbit with the disturbance from Earth's gravitational attraction due to its ellipticity, represented by the zonal harmonic of degree 2 (J2), which is indicated as one of the most significant disturbance. We also tested and compared polynomial models. From the use of different platform models were performed several experiments with two PRISM-ALOS images. Based on a distribution of check points on the images, planimetric accuracies were calculated. Almost general, the estimated values of mean square error for the check points were very close in terms of GSD (Ground Sample Distance).

Keywords: Exterior orientation, PRISM-ALOS, Zonal Harmonic J2.

\section{Introdução}

A utilização de imagens obtidas por plataformas orbitais como fonte de extração de informações espaciais, construção e atualização de documentos cartográficos tem sido cada vez mais explorada. Dentro de um determinado limite de escala, algumas vantagens destas imagens em relação às fotografias aéreas convencionais podem ser evidenciadas, tais como: baixo custo, imageamento de grandes áreas e frequência de aquisição de dados e sensores com resolução espacial cada vez mais finas, abre possibilidades de utilização destes produtos com grande relevância em estudos de diversas áreas do conhecimento.

Neste contexto, as imagens provenientes do satélite japonês ALOS (Advanced Land Observation Satellite), lançado em 24 de janeiro de 2006 e fora de operação desde 21 de abril de 2011, devido a problemas ocorridos nos sistemas de geração de energia, tiveram o objetivo de contribuir para o mapeamento, observação da cobertura da terra, monitoramento de desastres e levantamento de recursos naturais (Japan Aerospace Exploration Agency - JAXA, 2010).

Particularmente para a Cartografia brasileira, as imagens do satélite ALOS foram amplamente utilizadas tanto em instituições públicas, federais, municipais e estaduais, bem como no setor privado. Parte deste resultado é atribuído ao acordo de cooperação entre o Instituto Brasileiro de Geografia e Estatística - IBGE e a Alaska Satellite Facility - ASF, que estabelecia o IBGE como responsável pela distribuição das imagens do sensor PRISM-ALOS para órgãos dos governos federal, estadual e municipal, instituições de pesquisa e demais usuários não comerciais do Brasil, a preços acessíveis. O acordo chegou ao fim em meados do ano de 2011, porém as imagens ainda encontram-se em utilização, sendo comercializadas amplamente no Brasil e no mundo.

Para que haja a possibilidade de extração de informações geodésicas e/ou cartográficas destas imagens é necessária que seja realizada, além da correção radiométrica, a orientação exterior, isto é, a definição das posições do Centro Perspectivo (CP) do sensor e seus ângulos de orientação durante a formação da cena; e a orientação interior do sensor, a qual permite recuperar a geometria de imageamento. Atualmente os modelos matemáticos para orientação de imagens de varredura linear orbitais são definidos de acordo com a complexidade, exatidão e aplicabilidade, em duas classes, a saber (Toutin, 2004): modelos rigorosos, em geral, baseados no princípio da Colinearidade; e modelos ditos generalizados, baseando-se no uso de expressões polinomiais.

Os modelos rigorosos são indicados pela literatura como os que apresentam resultados potencialmente mais acurados (Jacobsen, 2007). Tais modelos objetivam reproduzir o processo físico de formação da cena, levando em conta as informações de geometria interna e externa do 
sensor e utilizando, em geral, o princípio de colinearidade com pontos. Outra alternativa é o modelo de coplanaridade com retas (Marcato junior e Tommaselli, 2013). Uma vez que estes princípios para sensores de varredura linear são adaptados à natureza dinâmica da formação da cena, deve haver uma preocupação quanto à modelagem das mudanças dos POE durante o processo de geração da cena, ou seja, um modelo matemático que melhor descreva as variações da posição e orientação da plataforma, durante o tempo de aquisição da cena.

Ao efetuar uma análise sobre os modelos que utilizam polinômios de $2^{\circ}$ grau para modelar a órbita, durante o tempo de imageamento, Michalis (2005) associou os termos lineares com a velocidade da plataforma e os termos quadráticos com as acelerações. A partir desta consideração, estes mesmos autores desenvolveram um modelo de plataforma denominado Modelo de Kepler, uma vez que as acelerações são estimadas a partir da solução do Problema dos Dois Corpos (Movimento Kepleriano).

No entanto, mesmo um arco de órbita pequeno e bem controlado como as de satélites de Sensoriamento Remoto apresentam influências de diversas perturbações, principalmente a atração devido ao potencial gravitacional terrestre e o arrasto atmosférico (Seeber, 2003). Segundo Light et al. (1980), algumas das causas de distorções em imagens orbitais, relacionadas à plataforma e à Terra são: movimento não elíptico da plataforma (órbita perturbada); influência da gravidade, da forma e do movimento da Terra. Desta forma, tais influências representam erros sistemáticos e devem ser consideradas na propagação dos POE.

Outro ponto também a ser considerado é que no Modelo de Kepler os pontos de apoio e verificação devem estar no Sistema de Coordenadas Geocêntrico Cartesiano Inercial (Michalis, 2005), o que implica em mais uma atividade a ser realizada para o uso deste modelo. Uma forma de contornar esta questão é utilizar o Problema dos Dois Corpos no Sistema de Coordenadas Geocêntrico Cartesiano Terrestre, acrescentando termos em função da velocidade angular de rotação da Terra conforme apresentado em Leick (2004). A desconsideração das componentes de Precessão e Nutação não causam prejuízo neste caso, tendo em vista que o intervalo de obtenção das cenas PRISM/ALOS é relativamente pequeno ( 6s).

Diante do apresentado, objetiva-se investigar o efeito da inserção de termos adicionais na aceleração proposta por Michalis (2005), no Modelo de Kepler, que representem as perturbações na órbita, bem como a parcela que faça com que o Problema dos Dois Corpos seja utilizado com coordenadas do Sistema de Coordenadas Geocêntrico Cartesiano Terrestre. Considerando que uma das perturbações orbitais mais significativas é a atração gravitacional terrestre devido a sua elipticidade, representada pelo harmônico zonal $\mathrm{J}_{2}$, somente esta pode ser levada em consideração. Outra questão é que, conforme citado, o intervalo de obtenção das cenas é pequeno, e, neste caso, as demais perturbações orbitais não apresentam influências significativas.

Apesar dos testes nesta pesquisa terem sido realizados com cenas PRISM do satélite ALOS, o qual se encontra fora de operação, as metodologias investigadas podem ser aplicadas em outros sensores de varredura linear orbitais e em imagens PRISM que ainda estejam sendo utilizadas.

\section{O Satélite ALOS e o sensor PRISM}

O satélite ALOS foi lançado pela JAXA, em 24 de janeiro de 2006, do centro espacial de Tanegashima (Japão). Sua órbita era circular heliossíncrona com altitude de $691,65 \mathrm{~km}$ (do equador) e inclinação de $98,16^{\circ}$. Como objetivos principais tinha-se a geração de dados cartográficos globais, monitoramento de recursos ambientais e naturais mundiais, monitoramento 
de grandes desastres em escala global e desenvolvimento de tecnologias para o futuro da observação da Terra (JAXA, 2010).

Um dos sensores instalado a bordo do ALOS era o sensor PRISM, composto por três sistemas ópticos independentes, permitindo o imageamento simultâneo nas direções vante (forward), nadir e ré (backward), podendo proporcionar visão estereoscópica e produção de Modelos Digitais de Superfície (MDS). As inclinações nominais das visadas vante e ré em relação ao nadir são de $\pm 23,8$ graus para ter razão base/altura de 1,0. A Figura 1 ilustra a composição do sensor.

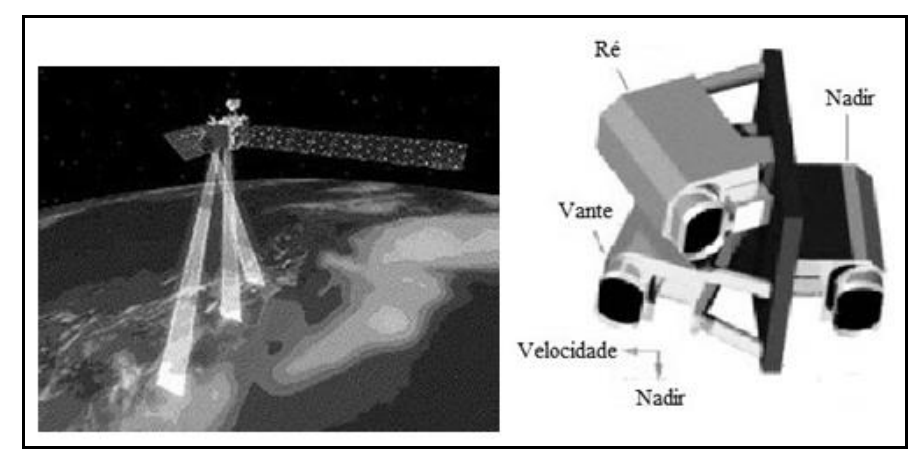

Figura 1: Geometria de funcionamento do sensor PRISM.

Fonte: Adaptado de JAXA (2010).

Algumas principais características do sensor PRISM e alguns dos parâmetros de orientação interior (POI) são apresentados na Tabela 1.

Tabela 1: Características e Parâmetros de orientação interior (POI) do sensor PRISM-ALOS.

\begin{tabular}{c|c} 
Características & POI \\
\hline Faixa espectral $0,52-0,77 \mu \mathrm{m}$ & Distância focal $1939 \mathrm{~mm}$ \\
\hline Resolução radiométrica $8 \mathrm{bits}$ & Tamanho do pixel no elemento CCD \\
\hline Elementos de resolução espacial $2,5 \mathrm{~m}$ (nadir) & $\mathrm{N}^{\circ}$ de colunas do elemento CCD \\
\hline Largura da faixa imageada & 14496 \\
$70 \mathrm{~km}$ nadir e 35 km no modo tripleto & Tempo de integração da linha CCD \\
\hline
\end{tabular}

Fonte: JAXA (2010)

As imagens do sensor PRISM são oferecidas com diversos níveis de processamento geométrico/radiométrico, a saber: o nível 1A, 1B1, 1B2-R e o nível 1B2G. Para utilização de modelos rigorosos devem ser utilizados os níveis 1A ou 1B1, uma vez que a imagem deve estar sem reamostragem e sem projeção cartográfica que altere as condições geométricas originais de imageamento, a serem modeladas. Mais detalhes sobre os níveis podem ser vistos em JAXA (2010). 


\section{Movimento kepleriano e perturbações orbitais}

A equação de movimento de um satélite artificial em torno de uma Terra esférica, sujeito apenas ao campo gravitacional desta é dada a partir da solução do Problema dos Dois Corpos (Bate et al., 1971; Seeber, 2003), no qual o vetor aceleração do satélite é dado por:

$$
\overrightarrow{\vec{r}}=-\frac{G M}{r^{g}} \vec{r}
$$

onde $\ddot{\vec{r}}$ e $\vec{r}$ são respectivamente o vetor aceleração e posição da plataforma no Sistema de Coordenadas Geocêntrico Cartesiano Inercial, $M$ é a massa da Terra e $G$ é a constante gravitacional universal $\left(6,673 \times 10^{-11} \mathrm{~m}^{3} / \mathrm{Kg} . \mathrm{s}^{2}\right)$, de forma que por exemplo para o sistema de referência WGS84, $G M=3,9860047 \times 10^{14} \mathrm{~m}^{3} \mathrm{~s}^{-2}$.

A Equação (1) também é conhecida como Equação do Movimento Kepleriano. Este movimento é considerado como uma primeira aproximação da órbita verdadeira do satélite, uma vez que não considera outras acelerações conhecidas como perturbações orbitais (BATE et al., 1971). Estas acelerações orbitais são forças atuantes de outras naturezas sobre o satélite.

Segundo Seeber (2003), entre os principais efeitos que fazem com que a órbita altere-se com o tempo estão a não esfericidade e não homogeneidade na distribuição da massa da Terra, além do seu achatamento, e também efeitos decorrentes do arrasto atmosférico, pressão de radiação solar e perturbações gravitacionais de outros corpos, notadamente da Lua e do Sol. Devido às estas perturbações, o movimento do satélite fica sujeito a acelerações adicionais. Deste modo, a Equação (1) torna-se (Seeber, 2003):

$$
\ddot{\vec{r}}=-\frac{G M}{r^{a}} \vec{r}+\vec{A}
$$

onde $\vec{A}$ representa “todas” as acelerações perturbadoras que atuam sobre o satélite, ou seja:

$$
\vec{A}=\overrightarrow{A_{G}}+\overrightarrow{A_{S L}}+\overrightarrow{A_{P R}}+\overrightarrow{A_{A L}}+\overrightarrow{A_{M}}+\overrightarrow{A_{A}}
$$

onde $\overrightarrow{A_{G}}$ é aceleração devido à atração gravitacional terrestre; $\overrightarrow{A_{S L}}$ é a aceleração devido à atração gravitacional direta do Sol e da Lua; $\overrightarrow{A_{p R}}$ é a aceleração devido à pressão de radiação solar; $\overrightarrow{A_{A L}}$ é a aceleração devido ao albedo ou pressão de radiação solar indireta; $\overrightarrow{A_{M}}$ é a aceleração devido à atração gravitacional indireta do Sol e da Lua, ou marés e; $\overrightarrow{A_{A}}$ é a aceleração devido ao arrasto atmosférico.

\subsection{Campo gravitacional da Terra}

De acordo com Leick (2004), o vetor aceleração devido ao campo gravitacional da Terra real é dado por: 


$$
\overrightarrow{A_{G}}=\left[\begin{array}{lll}
\frac{\partial V}{\partial x_{I}} & \frac{\partial V}{\partial Y_{I}} & \frac{\partial V}{\partial z_{I}}
\end{array}\right]^{T}
$$

onde $V$ é o potencial gravitacional e $X_{I}, Y_{I}$ e $Z_{I}$ são coordenadas cartesianas geocêntricas inerciais. Uma vez que $V$ é uma função harmônica no exterior das massas planetárias, esta pode ser desenvolvida em uma expansão em série de harmônicos esféricos (Torgue, 2001):

$$
V=\frac{G M}{r}\left[1+\sum_{n=0}^{\infty} \sum_{m=0}^{n}\left(\frac{a_{\theta}}{r}\right)^{n}\left(C_{n m} \cos m \lambda+S_{n m} \operatorname{sen} m \lambda\right) P_{n m}(\cos \vartheta)\right]
$$

onde $P_{n m}$ é a Função Associada de Legendre, dada por:

$$
P_{n m}(t)=\frac{1}{2^{n} n !}(1+t)^{\frac{m}{2}} \frac{d^{n+m}}{d t^{n+m}}\left(t^{2}-1\right)^{n}
$$

com:

$$
t=\operatorname{sen} \vartheta
$$

onde $C_{n m}$ e $S_{n m}$ são os coeficientes dos harmônicos esféricos do potencial $V ; a$ é o semi-eixo maior do elipsóide de referência adotado; $\vartheta$ e $\lambda$ são a co-latitude e a longitude geocêntricas, referentes a uma esfera de mesmo volume que o Elipsóide de Referência adotado.

Os coeficientes dos harmônicos esféricos do potencial estão relacionados às deformações que a Terra apresenta em relação a uma Terra perfeitamente esférica (Seeber, 2003). Deste modo, o coeficiente $\mathrm{C}_{00}=1$, representa a Terra com distribuição esférica de massa, e, para a origem do Sistema de Coordenadas coincidindo com o centro de massa da Terra, tem-se: $\mathrm{C}_{10}=\mathrm{C}_{11}=\mathrm{S}_{11}=0$ e $S_{00}=S_{n 0}=0$. Deste modo, a Equação (5) torna-se:

$$
V=\frac{G M}{r}\left[1+\sum_{n=2}^{\infty} \sum_{m=0}^{n}\left(\frac{a}{r}\right)^{n}\left(C_{n m} \cos m \lambda+S_{n m} \operatorname{sen} m \lambda\right) P_{n m}(\cos \vartheta)\right]
$$

Segundo Bate et al. (1971), Seeber (2003) e ainda Leick (2004), o coeficiente zonal $\mathrm{C}_{20}=-\mathrm{J}_{2}$ representa o efeito do achatamento da Terra no campo gravitacional, de modo que sua magnitude é aproximadamente 1000 vezes maior que os demais coeficientes dos harmônicos esféricos, o que o torna potencialmente mais significativo na determinação da aceleração devido à atração gravitacional terrestre. Ainda de acordo com Seeber (2003), de longe, a perturbação dominante em satélites de órbitas próximas da Terra é devido ao achatamento da mesma.

Utilizando então $\mathrm{n}=2$ e $\mathrm{m}=0$, a Equação (8) em função de coordenadas cartesianas geocêntricas inerciais torna-se:

$$
V=\frac{G M \cdot a_{e}^{2}}{r^{3}} J_{2}\left(\frac{3}{2} \frac{z^{2}}{r^{2}}-\frac{1}{2}\right)
$$


de forma que as componentes do vetor aceleração na Equação (4) levando em conta apenas o coeficiente zonal $\mathrm{J}_{2}$ pode ser obtida por:

$$
\begin{aligned}
& \ddot{X}_{I}=-\frac{G M}{r_{I}^{\mathrm{a}}} X_{I}-\frac{3}{2} J_{2} \frac{G M a_{g}^{2}}{r_{I}^{5}} X_{I}\left(1-5 \frac{z_{I}^{2}}{r_{I}^{2}}\right), \\
& \ddot{Y}_{I}=-\frac{G M}{r_{I}^{\mathrm{s}}} Y_{I}-\frac{3}{2} J_{2} \frac{G M a_{e}^{2}}{r_{I}^{5}} Y_{I}\left(1-5 \frac{z_{I}^{2}}{r_{I}^{2}}\right), \\
& \ddot{Z}_{I}=-\frac{G M}{r_{I}^{\mathrm{g}}} Z_{I}-\frac{3}{2} J_{2} \frac{G M a_{g}^{2}}{r_{I}^{5}} Z_{I}\left(1-5 \frac{z_{I}^{2}}{r_{I}^{2}}\right),
\end{aligned}
$$

com:

$$
r_{I}=\sqrt{X_{I}^{2}+Y_{I}^{2}+Z_{I}^{2}}
$$

onde $X_{I}, Y_{I}, Z_{I}$, são as componentes da posição no Sistema de Coordenadas Geocêntrico Cartesiano Inercial. Para se trabalhar com coordenadas no Sistema de Coordenadas Geocêntrico Cartesiano Terrestre, deve-se acrescentar nas Equações (10) termos adicionais em função da velocidade angular de rotação da Terra $\Omega_{\mathrm{t}}$. Desta forma tem-se (Leick, 2004):

$$
\begin{aligned}
& \ddot{X}=-\frac{G M}{r^{\mathrm{a}}} X-\frac{3}{2} J_{2} \frac{G M a_{\theta}{ }^{2}}{r^{5}} X\left(1-5 \frac{z^{2}}{r^{2}}\right)+\Omega_{t}^{2} X+2 \Omega_{t} \dot{Y}, \\
& \ddot{Y}=-\frac{G M}{r^{3}} Y-\frac{3}{2} J_{2} \frac{G M a_{\theta}{ }^{2}}{r^{5}} Y\left(1-5 \frac{z^{2}}{r^{2}}\right)+\Omega_{t}^{2} Y+2 \Omega_{t} \dot{X}, \\
& \ddot{Z}=-\frac{G M}{r^{3}} Z-\frac{3}{2} J_{2} \frac{G M a_{g}^{2}}{r^{5}} Z\left(1-5 \frac{Z^{2}}{r^{2}}\right) \text {. }
\end{aligned}
$$

com:

$$
r=\sqrt{X^{2}+Y^{2}+Z^{2}}
$$

onde $X, Y, Z, \dot{X}, \dot{Y}$ e $\dot{Z}$ são respectivamente as componentes de posição e velocidade no Sistema de Coordenadas Geocêntrico Cartesiano Terrestre.

\section{Orientação de imagens de varredura linear}

Os modelos rigorosos para orientação de imagens geralmente são baseados no princípio de colinearidade ou de coplanaridade, utilizando retas ou pontos. No caso do princípio de colinearidade, tem-se que pontos no espaço imagem, espaço objeto e o $\mathrm{CP}$ da câmera são colineares. Este princípio é modelado através das Equações de Colinearidade, que, no caso de sensores de varredura linear, são modificadas, incluindo a modelagem das mudanças nos POE em função do tempo ou da coordenada $x_{s}$ do Sistema de Coordenadas do sensor (Gugan e Dowman, 1988; Orun e Natarajan, 1994).

Uma vez que cada linha corresponde a uma imagem diferente, a componente $x_{s}$ do espaço imagem é assumida como zero, uma vez que não há deslocamentos nesta direção (Gugan e Dowman, 1988). A Equação de Colinearidade modificada para sensores de varredura linear é apresentada a seguir: 


$$
\begin{aligned}
& 0=x_{\mathrm{s}}=-f \frac{r_{11}\left(X_{\mathrm{i}}-X_{S}\right)+r_{12}\left(Y_{\mathrm{i}}-Y_{S}\right)+r_{18}\left(z_{\mathrm{i}}-z_{S}\right)}{r_{\mathrm{gn}}\left(X_{\mathrm{i}}-X_{S}\right)+r_{\mathrm{sg}}\left(Y_{\mathrm{i}}-Y_{S}\right)+r_{\mathrm{sg}}\left(z_{\mathrm{i}}-z_{S}\right)},
\end{aligned}
$$

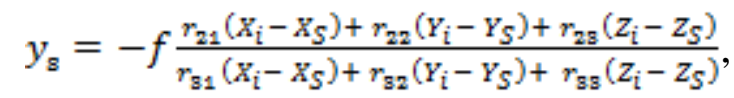

sendo $X_{i}, Y_{i}$ e $Z_{i}$ as coordenadas do ponto no espaço objeto; $X_{S}, Y_{S}$ e $Z_{S}$ as coordenadas do CP do sensor no Sistema de Referencia Terrestre (SRT) num dado instante de tomada da linha com o ponto de coordenadas $X_{i}, Y_{i}$ e $Z_{i} ; x_{s}, y_{s}$ são as coordenadas do Sistema de Referência do Sensor (SRS), sendo $x_{S}$ na direção de deslocamento do satélite; $f$ é a distância focal nominal do sensor e; $r_{11}, \ldots, r_{33}$ são os componentes da matriz de rotação. Esta última tem o objetivo de alinhar o Sistema de Coordenadas do espaço imagem com o Sistema de Coordenadas do espaço objeto.

Mesmo partindo do princípio de colinearidade, de uma forma geral, os modelos rigorosos podem ser separados em duas classes, a saber: modelos Posição-Rotação e modelos Órbita-Atitude (Kim e Dowman, 2006).

Nos modelos órbita-atitude, para o alinhamento do sistema de referência do espaço imagem com o sistema de referência do espaço objeto são considerados dois sistemas de referência intermediários. Estes são: o Sistema de Referência da Plataforma (SRP) e o Sistema de Referência Orbital (SRO). Deste modo, são utilizados então o SRS, o SRP, o SRO e o SRT. Uma vez que são considerados todos estes sistemas de referência, os dados de órbita disponibilizados nos arquivos auxiliares das imagens, como posição e velocidade, podem ser utilizados. No caso desta modelagem, os ângulos de atitude da plataforma $(R, P, \Psi)$ apresentam significado físico, pois alinham diretamente o SRP com o SRO. Mais detalhes sobre a estrutura e aplicações deste modelo podem ser vistos em Radhadevi et al. (1994), Kim e Dowman (2006) e Marcato Júnior e Tommaselli (2013).

Os modelos posição-rotação são os modelos primeiramente desenvolvidos e mais utilizados nos aplicativos comerciais. A diferença entre este modelo e o modelo órbita-atitude é que para o alinhamento do sistema de referencia do espaço imagem com o do espaço objeto são considerados apenas o SRS e o SRT. Deste modo, apenas uma matriz de rotação é definida, sendo obtida em função de ângulos de atitude sem significado físico, denominados ômega, phi e kappa $(\omega, \varphi, \kappa)$. Esta característica impede que os dados de órbita provenientes dos metadados das imagens $(R, P, \psi)$ sejam utilizados diretamente neste modelo, a não ser que haja a priori a transformação indicada no item anterior.

Para o caso dos modelos posição-rotação, diversas abordagens distintas foram desenvolvidas para a modelagem das variações dos POE durante a formação da cena. O modelo de plataforma desenvolvido por Gugan e Dowman (1988) apresenta o relacionamento entre os parâmetros de orientação exterior e o tempo $t$ modelado da seguinte forma:

$$
\begin{aligned}
X_{s} & =X_{0}+a_{1} t+b_{1} t^{2}, \\
Y_{s} & =Y_{0}+a_{2} t+b_{2} t^{2}, \\
Z_{s} & =Z_{0}+a_{3} t+b_{3} t^{2}, \\
\omega & =\omega_{0}+a_{4} t+b_{4} t^{2}, \\
\varphi & =\varphi_{0}+a_{5} t+b_{5} t^{2}, \\
\kappa & =\kappa_{0}+a_{6} t+b_{6} t^{2},
\end{aligned}
$$


onde $X_{0}, Y_{0}$ e $Z_{0}$ são as coordenadas do centro perspectivo do sensor na primeira linha da imagem; $a_{1}, a_{2}, \ldots, b_{5}, b_{6}$ são os coeficientes polinomiais da modelagem de mudança nos POE e; $t$ é um dado instante de tempo da formação da cena.

Entretanto, Orun e Natarajan (1994) propuseram um conjunto reduzido partindo das Equações (16), indicando que pequenas variações em $\omega$ tem o mesmo efeito que pequenas variações em $Y_{s}$ e; pequenas variações em $\varphi$ assemelham-se muito à pequenas variações em $X_{s}$. Neste caso, os autores indicaram que há a necessidade de se considerar como constante $\omega$ ou $Y$ e $\varphi$ ou $X$ do conjunto de parâmetros. Considerando que a estimativa da diferença de tempo $(t)$ é função linear da linha da imagem $(L)$, pode-se admitir que $L$ substitui a variação de tempo $t$, e as mudanças em unidades (fator de escala entre $t$ e $L$ ) podem ser absorvidas pelos coeficientes $a$ e $b$. Desta forma, as Equações (16) podem ser reescritas como:

$$
\begin{aligned}
& X_{s}=X_{0}+a_{1} L+b_{1} L^{2}, \\
& Y_{s}=Y_{0}+a_{2} L+b_{2} L^{2}, \\
& Z_{s}=Z_{0}+a_{3} L+b_{3} L^{2}, \\
& \kappa=\kappa_{0}+a_{4} L+b_{4} L^{2} .
\end{aligned}
$$

Diferentes configurações de modelos de plataforma em função da linha da imagem, considerando as injunções absolutas ou não em $\omega$ e $\varphi$ podem ser montadas. Exemplos podem ser vistos em Salamonowicz (1986), Gugan e Dowman (1988), Radhadevi et al. (1994), Bang e Cho (2001), Kim e Dowman (2006), Poli (2007), Jeong e Bethel (2008), Weser et al. (2008), Marcato Junior et al. (2011) e Rodrigues e Ferreira (2013).

\subsection{Modelo de Kepler}

Considerando que no caso dos modelos posição-rotação a propagação da posição do satélite, no modelo de plataforma, pode ser modelada a partir de um polinômio de segundo grau, Michalis (2005) apresentou a ideia de que os coeficientes lineares podem representar a velocidade da plataforma e os termos quadráticos as acelerações.

A partir destas considerações, as acelerações utilizadas nos polinômios de segundo grau podem ser calculadas a partir da Equação do Movimento Kepleriano e as equações do modelo da plataforma, associadas a teoria do Movimento uniformemente variado (MUV) são dadas por:

$$
\begin{aligned}
& X_{c}(t)=X_{0}+u_{x} \tau-\frac{G M \cdot X_{0} \cdot \tau^{2}}{2 \cdot\left(X_{0}{ }^{2}+Y_{0}{ }^{2}+z_{0}{ }^{2}\right)^{8 / 2}}, \\
& Y_{c}(t)=Y_{0}+u_{y} \tau-\frac{G M \cdot Y_{0} \cdot \tau^{2}}{2 \cdot\left(X_{0}{ }^{2}+Y_{0}{ }^{2}+Z_{0}{ }^{2}\right)^{8 / 2}}, \\
& Z_{c}(t)=Z_{0}+u_{z} \tau-\frac{G M \cdot Z_{0} \cdot \tau^{2}}{2 \cdot\left({X_{0}}^{2}+Y_{0}{ }^{2}+Z_{0}{ }^{2}\right)^{8 / 2}},
\end{aligned}
$$

onde $u_{x}, u_{y}, u_{y}$ são as componentes do vetor velocidade; $\tau$ é o produto entre $L$ e $d t$ que é o tempo de integração da linha CCD do sensor PRISM (Tabela 1). Para a utilização destes modelos, 
algumas considerações e indicações são apresentadas conforme descrito em Michalis e Dowman (2008):

- o satélite move-se ao longo de uma órbita bem definida e aproximadamente circular;

- as cenas são adquiridas com um intervalo de tempo constante de aquisição das linhas. Como resultado as coordenadas ao longo do trajeto da plataforma têm a mesma escala;

- a orientação das imagens deve ser realizada em um Sistema de Coordenadas Geocêntrico, a fim de evitar distorções causadas pela curvatura da Terra e da projeção cartográfica e para facilitar a integração com fontes de informações de metadados que podem estar disponíveis;

- o movimento do satélite durante o tempo de aquisição de imagens estéreo alongtrack é considerado um movimento Kepleriano.

Exemplos de aplicação deste modelo podem ser vistos em Dowman e Michalis (2003) utilizando cenas ASTER; Michalis e Dowman (2004), Michalis (2005), Michalis e Dowman (2008a) com cenas HRS-SPOT 5; com cenas do satélite CARTOSAT-I em Michalis e Dowman (2008b); Dowman et al (2010), Michalis e Dowman (2010) em cenas PRISM-ALOS e; em Rodrigues e Ferreira (2013) utilizando cenas HRC-CBERS 2B.

\section{Metodologia}

\subsection{Modelos para a orientação das imagens}

Nesta pesquisa, oito modelos de plataforma foram construídos e analisados. Uma vez que o ângulo $\kappa$ sofre variações devido ao movimento lateral do satélite (crab movement) de acordo com o argumento da latitude da órbita, para compensar o movimento de rotação da Terra (Satoru e Akihiro, 2011), investigou-se a influência de sua variação de forma linear e quadrática. Os ângulos $\omega$ e $\varphi$ foram considerados sem variações por serem bem controlados (acurácia de 1,08”) durante o tempo de formação das cenas (Satoru e Akihiro, 2011).

Para adaptar os modelos que utilizam coordenadas geocêntricas cartesianas inerciais ao uso das coordenadas geocêntricas cartesianas terrestres, as equações dos modelos da plataforma referentes às componentes das posições do satélite foram adaptadas, utilizando-se a teoria apresentada no sub-item 3.1 nas Equações (12) e (13). Nos modelos de $1 \mathrm{a} 4 \mathrm{o}$ parâmetro $d t$ utilizado para o cálculo de $\tau$ é considerado constante durante todo o tempo de formação das cenas.

O modelo 1, denominado aqui de K1 é o modelo de Kepler, considerando os ângulos de atitude $\varphi$ e $\omega$ invariáveis durante o tempo de aquisição das imagens. $O$ ângulo $\kappa$ é considerado variável e propagado com polinômio de $1^{\circ}$ grau. Os ângulos de atitude $\omega$ e $\varphi$, bem como o termo associado à variação do ângulo $\kappa$ são estimados no ajustamento de observações. Tem-se então:

$$
\begin{aligned}
& X_{s}(t)=X_{0}+u_{x} t+\left[-\frac{G M \cdot X_{0}}{2 \cdot r^{a}}+\Omega_{t}^{2} X_{0}+2 \Omega_{t} u_{y}\right] \cdot t^{2}, \\
& Y_{s}(t)=Y_{0}+u_{y} t+\left[-\frac{G M \cdot Y_{0}}{2 \cdot r^{3}}+\Omega_{t}^{2} Y_{0}+2 \Omega_{t} u_{x}\right] \cdot t^{2},
\end{aligned}
$$

Bol. Ciênc. Geod., sec. Artigos, Curitiba, v. 21, no 2, p.409-432, abr-jun, 2015. 


$$
\begin{gathered}
Z_{s}(t)=Z_{0}+u_{z} t-\frac{G M \cdot Z_{0}}{2 \cdot r^{3}} \times t^{2}, \\
\omega=\omega_{0} \\
\varphi=\varphi_{0} \\
\kappa=\kappa_{0}+d_{1} \times t .
\end{gathered}
$$

O modelo 2 foi denominado K2, sendo exatamente o modelo de Kepler, considerando os ângulos de atitude $\varphi$ e $\omega$ invariáveis durante o tempo de aquisição das imagens e $\kappa$ variável. Porém, o que o diferencia do anterior é o fato do ângulo $\kappa$ ser propagado a partir de um polinômio de $2^{\circ}$ grau, ou seja:

$$
\kappa=\kappa_{0}+d_{1} t+d_{2} t^{2}
$$

O Modelo 3 é o modelo de plataforma desenvolvido nesta pesquisa, denominado Modelo de órbita perturbada J2, e para os experimentos é denominado J2-1. A posição da plataforma é propagada a partir das Equações de Movimento Kepleriano, adicionando-se as acelerações devido à atração gravitacional terrestre, levando em consideração apenas o coeficiente $\mathbf{J}_{2}$ dos harmônicos esféricos do geopotencial. Nesta primeira configuração, consideram-se os ângulos de atitude $\varphi$ e $\omega$ invariáveis durante o tempo de aquisição das cenas, e o ângulo $\kappa$ é considerado variável e propagado com polinômio de $1^{\circ}$ grau. Os ângulos de atitude e a variação do ângulo $\kappa$ são estimados no processo de ajustamento de observações. O modelo de plataforma assume a forma:

$$
\begin{gathered}
X_{s}(t)=X_{0}+u_{x} t+\left[-\frac{G M}{r^{3}} X_{0}-\frac{3}{2} J_{2} \frac{G M a_{\varepsilon}^{2}}{r^{5}} X_{0} \times\left(1-5 \frac{z_{0}{ }^{2}}{r^{2}}\right)+\Omega_{t}{ }^{2} X_{0}+2 \Omega_{t} u_{y}\right] \cdot t^{2}, \\
Y_{s}(t)=Y_{0}+u_{y} t+\left[-\frac{G M}{r^{s}} Y_{0}-\frac{3}{2} J_{2} \frac{G M a_{\theta}^{2}}{r^{5}} Y_{0} \times\left(1-5 \frac{z_{0}{ }^{2}}{r^{2}}\right)+\Omega_{t}{ }^{2} Y_{0}+2 \Omega_{t} u_{x}\right] \cdot t^{2}, \\
Z_{s}(t)=Z_{0}+u_{z} t+\left[-\frac{G M}{r^{3}} Z_{0}-\frac{3}{2} J_{2} \frac{G M a_{\theta}^{2}}{r^{5}} Z_{0} \times\left(1-5 \frac{z_{0}^{2}}{r^{2}}\right)\right] \cdot t^{2}, \\
\omega=\omega_{0}, \\
\varphi=\varphi_{0}, \\
\kappa=\kappa_{0}+d_{1} \times \tau,
\end{gathered}
$$

onde:

$$
r=\sqrt{X_{0}^{2}+Y_{0}^{2}+Z_{0}^{2}}
$$

Uma vez que as imagens estão referenciadas ao ITRF97 (GRS80), os valores das constantes são $G M=3986005 \times 10^{8} \mathrm{~m}^{3} \mathrm{~s}^{-2}, a_{e}=6378137 \mathrm{~m}, \Omega_{t}=7292115 \times 10^{-11} \mathrm{rad} \mathrm{s}^{-1} \mathrm{e} I_{2}=1,08263 \times 10^{-3}$.

O modelo 4 aqui denominado de J2-2, é similar ao modelo 3, porém considerando que o ângulo $\kappa$ é variável e propagado utilizando-se polinômio de $2^{\circ}$ grau (Equação (20)).

O modelo 5, denominado aqui de ON1, é o modelo proposto por Orun e Natarajan (1994), o qual 
considera os ângulos de atitude $\omega$ e $\varphi$ invariáveis durante o tempo de aquisição das imagens (Equações (17)). Estes receberam injunções absolutas, assumindo respectivamente os valores de zero e zero para a câmera nadir, e de zero e $-23,8^{\circ}$ para a câmera ré. Para o modelo 5 , o ângulo $\kappa$ é considerado variável e propagado com polinômio de $1^{\circ}$ grau. $\mathrm{O}$ ângulo $\kappa$ e o termo associado à sua variação são estimados no ajustamento de observações.

O sexto modelo analisado (ON2) é o quinto modelo, porém considerando a variação do ângulo $\kappa$ de forma quadrática, ou seja:

$$
\kappa=\kappa_{0}+a_{4} L+b_{4} L^{2},
$$

O Modelo 7 (P1), considera a modelagem polinomial da trajetória, assim como o modelo proposto por Orun e Natarajan (1994), porém, o que o diferenciou deste último é a não aplicação das injunções absolutas nos ângulos $\varphi$ e $\omega$, ou seja, estes são estimados no ajustamento. Similarmente aos demais modelos, estes dois ângulos foram considerados invariáveis durante o tempo de aquisição das imagens. Neste modelo, o ângulo $\kappa$ é considerado variável e propagado com polinômio de $1^{\circ}$ grau.

O oitavo modelo testado (P2) é o sétimo modelo, porém considerando a variação do ângulo $\kappa$ de forma quadrática (Equação (20)).

O Sistema de Coordenadas do espaço objeto no caso dos modelos ON1, ON2, P1 e P2 foi o Sistema Geodésico Local, adotando como origem em cada cena a média das coordenadas dos pontos de apoio distribuídas sobre a mesma.

Para a estimativa dos parâmetros nos oito modelos, utilizou-se o Método dos Mínimos Quadrados com o método paramétrico (Gemael, 1994), tendo em vista que as equações dos modelos de orientação utilizados foram organizadas de maneira a isolar as observações como função dos parâmetros de forma explícita. Os parâmetros estimados nos ajustamentos em cada modelo são indicados na Tabela 2 a seguir.

Tabela 2: Parâmetros a serem estimados no ajustamento.

\begin{tabular}{c|c}
\hline Modelo de Plataforma & Parâmetros \\
\hline K1 e J2-1 & $X_{0}, Y_{0}, Z_{0}, u_{x}, u_{y}, u_{z}, \omega, \varphi, \kappa, d_{1}$. \\
\hline K2 e J2-2 & $X_{0}, Y_{0}, Z_{0}, u_{x}, u_{y}, u_{z}, \omega, \varphi, \kappa, d_{1}, d_{2}$. \\
\hline ON1 & $X_{0}, a_{1}, b_{1}, Y_{0}, a_{2}, b_{2}, Z_{0}, a_{3}, b_{3}, \kappa, a_{4}$. \\
\hline ON2 & $X_{0}, a_{1}, b_{1}, Y_{0}, a_{2}, b_{2}, Z_{0}, a_{3}, b_{3}, \kappa, a_{4}, b_{4}$. \\
\hline P1 & $X_{0}, a_{1}, b_{1}, Y_{0}, a_{2}, b_{2}, Z_{0}, a_{3}, b_{3}, \omega, \varphi, \kappa, a_{4}$. \\
\hline P2 & $X_{0}, a_{1}, b_{1}, Y_{0}, a_{2}, b_{2}, Z_{0}, a_{3}, b_{3}, \omega, \varphi, \kappa, a_{4}, b_{4}$. \\
\hline
\end{tabular}

\subsection{Estudos de caso}

Nesta pesquisa foram utilizadas duas imagens (15046/4100 e 15046/4045) pertencentes a um tripleto PRISM, de 20/11/2008, em nível de processamento 1B1. Estas imagens foram cedidas pelo Departamento de Cartografia da Faculdade de Ciências e Tecnologia da Universidade Estadual Paulista. A área de aproximadamente 35 x $35 \mathrm{~km}$ coberta por cada imagem abrange o município de Presidente Prudente - SP e região.

As coordenadas terrestres utilizadas, tanto no processo de orientação quanto no processo de 
verificação de acurácias, foram obtidas por (Toledo et al., 2010) a partir de rastreios com receptores GNSS (Global Navigation Satellite System), utilizando o método de posicionamento relativo estático com tempo de rastreio de, no mínimo, 30 minutos. Isto com a finalidade de atingir um erro menor que $0,5 \mathrm{~m}$, que corresponde a, aproximadamente, um terço do GSD (Ground Sample Distance). O processamento dos dados foi realizado com efemérides precisas no software TGO (Trimble Geometics Office).

Dois grupos de experimentos foram definidos baseados em diferentes quantidades de pontos de apoio e verificação. No primeiro grupo foram selecionados 14 pontos de apoio (PA) e 9 pontos de verificação (PV), sendo que em decorrências das duas cenas recobrirem quase o mesmo local, os pontos foram os mesmos paras ambas. No segundo grupo, as configurações foram invertidas, ou seja, 9 pontos de apoio (PA) e 14 de verificação. A Figura 2 apresenta a distribuição dos pontos de apoio e verificação nas duas cenas utilizadas nos dois grupos de experimentos. No espaço imagem, os pontos foram observados no aplicativo ERDAS/LPS 2010 de modo manual. Na pesquisa, a precisão considerada na medição foi de 1 pixel.

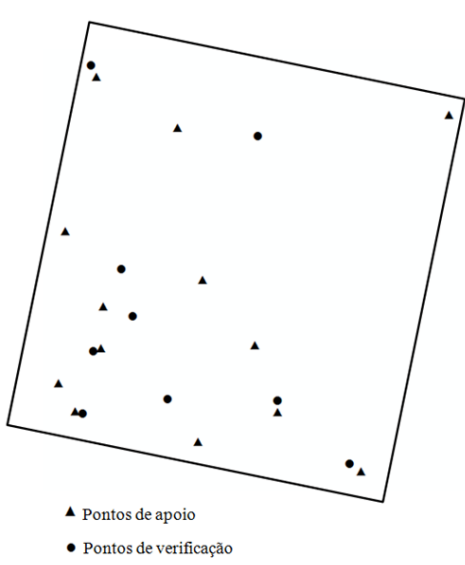

(a)

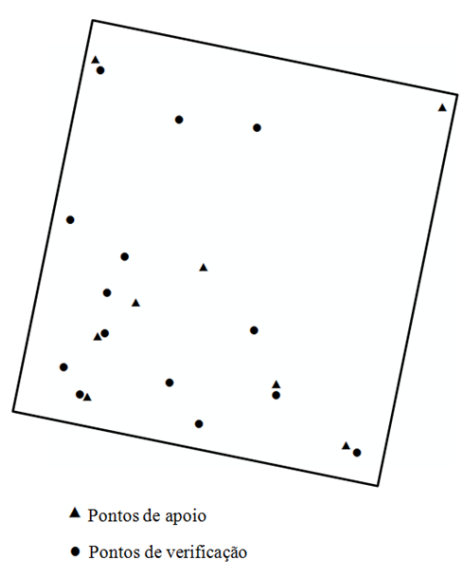

(b)

Figura 2: Distribuição dos pontos de apoio e verificação nas cenas do primeiro grupo de experimentos (a) e do segundo grupo de experimentos (b).

\section{Resultados}

\subsection{Resultados obtidos com a imagem em visada Ré}

Estes resultados referem-se à aplicação dos modelos apresentados no item 5.1 na orientação da imagem em visada ré, utilizando as duas configurações de pontos de apoio e verificação, sendo denominados experimentos de 1 a 16 respectivamente, conforme indicado na Tabela 3 a seguir.

Após o ajustamento das observações, a partir dos pontos de apoio selecionados no espaço objeto utilizando cada um dos modelos, foi possível estimar os POE das imagens e suas respectivas precisões a partir das Matrizes Variância-Covariância (MVCs). Em todos os experimentos, em ambos os casos de distribuição de pontos de apoio, as precisões dos POE apresentaram comportamento semelhante com relação às ordens de grandeza. $\mathrm{O}$ valor mais alto de precisão chegou a no máximo duas ordens de grandeza a menos do que o valor do seu respectivo 
parâmetro.

Tabela 3: Experimentos para a imagem em visada ré.

\begin{tabular}{cc|cc}
\hline \multicolumn{2}{c|}{ Configuração de pontos de apoio 1 } & \multicolumn{2}{c}{ Configuração de pontos de apoio 2 } \\
\hline Experimento & Modelo & Experimento & Modelo \\
\hline 1 & $\mathrm{~K} 1$ & 9 & $\mathrm{~K} 1$ \\
\hline 2 & $\mathrm{~K} 2$ & 10 & $\mathrm{~K} 2$ \\
\hline 3 & $\mathrm{~J} 2-1$ & 11 & $\mathrm{~J} 2-1$ \\
\hline 4 & $\mathrm{~J} 2-2$ & 12 & $\mathrm{~J} 2-2$ \\
\hline 5 & $\mathrm{ON} 1$ & 13 & $\mathrm{ON} 1$ \\
\hline 6 & $\mathrm{ON} 2$ & 14 & $\mathrm{ON} 2$ \\
\hline 7 & $\mathrm{P} 1$ & 15 & $\mathrm{P} 1$ \\
\hline 8 & $\mathrm{P} 2$ & 16 & $\mathrm{P} 2$ \\
\hline
\end{tabular}

Como anteriormente citado, o ângulo $\kappa$ sofre variações devido ao movimento lateral do satélite (crab movement), e, conforme indicado no item 3.2, foram verificadas variações lineares e quadráticas. Com relação à variação linear, para os experimentos $1,3,9$ e 11, os valores foram em média de aproximadamente 30" $/ \mathrm{s}$. Neste caso, a não consideração desta pode acarretar em pequenos deslocamentos nas coordenadas do espaço objeto ao longo da cena. Com relação à variação quadrática, para os experimentos 2, 4, 10 e 12, em média os valores ficaram em torno de alguns centésimos de " $/ s$, não sendo capaz de proporcionar deslocamentos significativos das cenas no caso de sua desconsideração. Esta afirmação pode ser comprovada a partir da Tabela 7 das raízes dos erros médios quadráticos (RMSE) dos pontos de verificação. Para os experimentos 5, 6, 13 e 14, tanto as variações lineares quanto as quadráticas apresentaram-se na ordem de grandeza de milésimos de "/linha. Para os experimentos 7, 8, 15 e 16, as variações lineares apresentaram-se na ordem de grandeza de milésimos de "/linha, e as variações quadráticas de 10" ${ }^{7}$ a $10^{-8}$ "/linha. De forma geral, as variações quadráticas de $\kappa$ não modificaram de forma significativa a acurácia das orientações, conforme pode ser visto mais adiante nos valores de RMSE dos pontos de verificação.

Outro grupo de resultados analisados foi o proveniente dos ajustamentos de observações, como o teste do Qui-quadrado para análise das variâncias a priori e posteriori, e os RMSE dos vetores dos resíduos (Tabela 4).

Tabela 4: Resultados obtidos nos ajustamentos para os experimentos da imagem em visada ré com a distribuição de pontos 1 e 2 .

\begin{tabular}{|c|c|c|c|}
\hline $\begin{array}{c}\text { Exp. (MP) com } \\
\text { distribuição de pontos } 1\end{array}$ & Resultados & $\begin{array}{c}\text { Exp. (MP) com } \\
\text { distribuição de pontos } 2\end{array}$ & Resultados \\
\hline $1(\mathrm{~K} 1)$ & $\begin{array}{c}\chi_{\text {calculado: }}^{2} 12,0894 \\
\chi_{\text {teórico }}^{2}(18 ; 0,975): 31,53 \\
\operatorname{RMSE} x_{s}: 0,0049 \mathrm{~mm} \\
\operatorname{RMSE} y_{s}: 0,0048 \mathrm{~mm}\end{array}$ & $9(\mathrm{~K} 1)$ & $\begin{array}{c}\chi_{\text {calculado: }}^{2} 9,0213 \\
\chi_{\text {teórico }}^{2}(8 ; 0,975): 17.53 \\
\operatorname{RMSE} x_{s}: 0,0073 \mathrm{~mm} \\
\operatorname{RMSE} y_{s}: 0,0019 \mathrm{~mm}\end{array}$ \\
\hline $2(\mathrm{~K} 2)$ & $\begin{array}{c}\chi_{\text {calculado: }}^{2} 11,6850 \\
\chi_{\text {teórico }}^{2}(17 ; 0,975): 30,19 \\
\operatorname{RMSE} x_{s}: 0,0051 \mathrm{~mm} \\
\operatorname{RMSE} y_{s}: 0,0044 \mathrm{~mm}\end{array}$ & $10(\mathrm{~K} 2)$ & $\begin{array}{c}\chi_{\text {calculado }}^{2} 9,0050 \\
\chi_{\text {teórico }}^{2}(7 ; 0,975): 16.01 \\
\operatorname{RMSE} x_{s}: 0,0072 \mathrm{~mm} \\
\operatorname{RMSE} y_{s}: 0,0021 \mathrm{~mm}\end{array}$ \\
\hline
\end{tabular}

Bol. Ciênc. Geod., sec. Artigos, Curitiba, v. 21, nº 2, p.409-432, abr-jun, 2015. 


\begin{tabular}{|c|c|c|c|}
\hline $3(\mathrm{~J} 2-1)$ & $\begin{array}{c}\chi_{\text {calculado: }}^{2} 9,2805 \\
\chi_{\text {teórico }}^{2}(18 ; 0,975): 31,53 \\
\operatorname{RMSE} x_{s}: 0,0041 \mathrm{~mm} \\
\operatorname{RMSE} y_{s}: 0,0044 \mathrm{~mm}\end{array}$ & 11 (J2-1) & $\begin{array}{c}\chi_{\text {calculado }}^{2}: 10,7892 \\
\chi_{\text {teórico }}^{2}(8 ; 0,975): 17.53 \\
\operatorname{RMSE~} x_{s}: 0,0079 \mathrm{~mm} \\
\operatorname{RMSE} y_{s}: 0,0024 \mathrm{~mm}\end{array}$ \\
\hline $4(\mathrm{~J} 2-2)$ & $\begin{array}{c}\chi_{\text {calculado: }}^{2}: 9,1444 \\
\chi_{\text {teórico }}^{2}(17 ; 0,975): 30,19 \\
\operatorname{RMSE~} x_{s}: 0,0041 \mathrm{~mm} \\
\operatorname{RMSE} y_{s}: 0,0043 \mathrm{~mm}\end{array}$ & $12(\mathrm{~J} 2-2)$ & $\begin{array}{c}\chi_{\text {calculado }}^{2}: 10,7891 \\
\chi_{\text {teórico }}^{2}(7 ; 0,975): 16.01 \\
\operatorname{RMSE~} x_{s}: 0,0079 \mathrm{~mm} \\
\operatorname{RMSE} y_{s}: 0,0025 \mathrm{~mm}\end{array}$ \\
\hline $5(\mathrm{ON} 1)$ & $\begin{array}{c}\chi_{\text {calculado }}^{2}: 14.1930 \\
\chi_{\text {teórico }}^{2}(17 ; 0,975): 30,19 \\
\operatorname{RMSE~} x_{s}: 0,0045 \mathrm{~mm} \\
\operatorname{RMSE} y_{s}: 0,0059 \mathrm{~mm}\end{array}$ & 13 (ON1) & $\begin{array}{c}\chi_{\text {calculado }}^{2}: 61,4200 \\
\chi_{\text {teórico }}^{2}(7 ; 0,975): 16.01 \\
\operatorname{RMSE} x_{s}: 0,0195 \mathrm{~mm} \\
\operatorname{RMSE} y_{s}: 0,0028 \mathrm{~mm}\end{array}$ \\
\hline $6(\mathrm{ON} 2)$ & $\begin{array}{c}\chi_{\text {calculado }}^{2}: 14,1468 \\
\chi_{\text {teórico }}^{2}(16 ; 0,975): 28,85 \\
\operatorname{RMSE~} x_{s}: 0,0044 \mathrm{~mm} \\
\operatorname{RMSE} y_{s}: 0,0059 \mathrm{~mm}\end{array}$ & 14 (ON2) & $\begin{array}{c}\chi_{\text {calculado }}^{2}: 61,2716 \\
\chi_{\text {teórico }}^{2}(6 ; 0,975): 14.45 \\
\operatorname{RMSE} x_{s}: 0,0195 \mathrm{~mm} \\
\operatorname{RMSE} y_{s}: 0,0028 \mathrm{~mm}\end{array}$ \\
\hline 7 (P1) & $\begin{array}{c}\chi_{\text {calculado: }}^{2}: 7,5052 \\
\chi_{\text {teórico }}^{2}(15 ; 0,975): 27,49 \\
\operatorname{RMSE} x_{s}: 0,0041 \mathrm{~mm} \\
\operatorname{RMSE} y_{s}: 0,0035 \mathrm{~mm}\end{array}$ & 15 (P1) & $\begin{array}{c}\chi_{\text {calculado }}^{2} 6,9935 \\
\chi_{\text {teórico }}^{2}(5 ; 0,975): 12.83 \\
\operatorname{RMSE} x_{s}: 0,0066 \mathrm{~mm} \\
\operatorname{RMSE} y_{s}: 0,0005 \mathrm{~mm}\end{array}$ \\
\hline 8 (P2) & $\begin{array}{c}\chi_{\text {calculado: }}^{2}: 7,4527 \\
\chi_{\text {teórico }}^{2}(14 ; 0,975): 26,12 \\
\operatorname{RMSE} x_{s}: 0,0041 \mathrm{~mm} \\
\operatorname{RMSE~} y_{s}: 0,0035 \mathrm{~mm}\end{array}$ & $16(\mathrm{P} 2)$ & $\begin{array}{c}\chi_{\text {calculado }}^{2}: 0,1802 \\
\chi_{\text {teórico }}^{2}(4 ; 0,975): 11,1433 \\
\operatorname{RMSE} x_{s}: 0,0009 \mathrm{~mm} \\
\operatorname{RMSE} y_{s}: 0,0005 \mathrm{~mm}\end{array}$ \\
\hline
\end{tabular}

Observa-se na Tabela 4 que com exceção dos experimentos 13 e 14, a hipótese básica de $\widehat{\sigma}_{0}^{2}=$ $\sigma_{0}^{2}\left(\right.$ com $\left.\sigma_{0}^{2}=1\right)$ não é rejeitada ao nível de significância de $5 \%$ no teste do Qui-quadrado. Nos resultados dos experimentos de 1 a 12 e de 15 e 16, as ordens de grandeza dos componentes dos vetores dos resíduos em $x_{s}$ e $y_{s}$ apresentaram-se adequadas, na maioria das vezes apresentando valores abaixo do pixel e na minoria das vezes chegando a apresentar valores em torno de 1 pixel. Nos experimentos 13 e 14 os valores dos resíduos foram mais elevados (apenas na componente $x_{s}$ ) em relação aos demais experimentos, chegando na grande maioria dos pontos a valores maiores do que 1 pixel, com valor máximo de $0,0371 \mathrm{~mm}$. Este fato levou à rejeição da hipótese básica no teste do Qui-quadrado. Isto pode ter sido causado por erros sistemáticos decorrentes da aplicação das injunções absolutas nos ângulos $\omega$ e $\varphi$, tendo em vista que nos experimentos 15 e 16, onde tais ângulos foram estimados nos ajustamentos, não houveram valores elevados de resíduo.

Embora a magnitude dos valores das componentes do vetor dos resíduos tenham sido diferentes, em todos os experimentos as médias dos mesmos foram próximas de zero $\left(10^{-6}\right.$ no máximo). Exceto para os experimentos 13 e 14 pode-se indicar que, considerando os testes na imagem em visada ré, os modelos matemáticos conseguiram representar satisfatoriamente a realidade física do processo. 
Posteriormente à estimativa dos parâmetros e aplicação dos mesmos no modelo de Colinearidade inverso, a avaliação da acurácia das orientações foi realizada. Nas Equações de Colinearidade inversa, para o valor de $Z_{i}$ foram utilizados os valores dos próprios pontos de verificação. Neste processo, as coordenadas dos pontos de verificação, foram transformadas de coordenadas planoretanguares do sistema UTM $(\mathrm{E}, \mathrm{N})$ para coordenadas do Sistema Geodésico Local $\left(\mathrm{X}_{\mathrm{L}}, \mathrm{Y}_{\mathrm{L}}\right)$, uma vez que este Sistema de Coordenadas permite uma representação mais fiel da situação dos deslocamentos na superfície terrestre. A Tabela 5 a seguir apresenta a acurácia nas componentes $\mathrm{X}_{\mathrm{L}}$ e $\mathrm{Y}_{\mathrm{L}}$ em todos os modelos analisados na imagem em visada ré.

Tabela 5: Acurácia nas componentes $X_{L}, Y_{L}$ para os pontos de verificação nos experimentos da imagem em visada ré.

\begin{tabular}{c|c|c|c|c|c}
\hline \multirow{2}{*}{$\begin{array}{c}\text { Exp. (Modelo) com } \\
\text { distribuição de pontos 1 }\end{array}$} & \multicolumn{2}{|c|}{ RMSE (m) } & $\begin{array}{c}\text { Exp. (Modelo) com } \\
\text { distribuição de pontos 2 }\end{array}$ & \multicolumn{2}{c}{$\mathbf{X}_{\mathbf{L}}$} \\
\hline $1(\mathrm{~K} 1)$ & 1,961 & 1,468 & $9(\mathrm{~K} 1)$ & 1,712 & 2,764 \\
\hline $2(\mathrm{~K} 2)$ & 1,948 & 1,502 & $10(\mathrm{~K} 2)$ & 1,718 & 2,725 \\
\hline $3(\mathrm{~J} 2-1)$ & 1,957 & 1,101 & $11(\mathrm{~J} 2-1)$ & 1,854 & 2,860 \\
\hline $4(\mathrm{~J} 2-2)$ & 1,990 & 1,116 & $12(\mathrm{~J} 2-2)$ & 1,862 & 2,856 \\
\hline $5(\mathrm{ON} 1)$ & 2,714 & 2,442 & $13(\mathrm{ON} 1)$ & 2,495 & 16,190 \\
\hline $6(\mathrm{ON} 2)$ & 2,711 & 2,386 & $14(\mathrm{ON} 2)$ & 2,468 & 16,051 \\
\hline $7(\mathrm{P} 1)$ & 1,834 & 2,350 & $15(\mathrm{P} 1)$ & 5,158 & 3,871 \\
\hline $8(\mathrm{P} 2)$ & 1,827 & 2,452 & $16(\mathrm{P} 2)$ & 5,124 & 4,666 \\
\hline
\end{tabular}

A partir da análise dos valores da Tabela 5 observa-se inicialmente que a consideração da variação quadrática do ângulo $\kappa$ não influenciou de maneira significativa na qualidade das orientações, apresentando-se por vezes ligeiramente melhor ou pior. Observa-se também que a adaptação dos modelos de plataforma nos experimentos de 1 a 4 e de 9 a 12 ao uso das coordenadas referenciadas ao Sistema de Coordenadas Geocêntricas Cartesianas Terrestres, apresentou-se eficaz, tendo em vista que os valores de RMSE alcançaram valores de em torno de 1 pixel ou menor. Em relação à inserção do termo que modela a perturbação orbital do satélite, proveniente da atração gravitacional terrestre devido a sua elipticidade, representada pelo harmônico zonal $\mathrm{J}_{2}$, tem-se que esta não alterou significativamente as acurácias das orientações, também se apresentando por vezes pouco melhor ou pior em reação aos demais modelos. Percebe-se ainda com os resultados da Tabela 5 que para o caso da imagem em visada ré houve uma menor flexibilidade de quantidade e distribuição de pontos de apoio nos modelos polinomiais com relação aos resultados de acurácia, e, que de forma geral, os experimentos que utilizaram os modelos ON1 e ON2 apresentaram piores resultados do que os experimentos utilizando os modelos P1 e P2. Um indicativo de tais resultados é a aplicação das injunções absolutas nos ângulos $\omega$ e $\varphi$ ao invés de estimá-los como parâmetros nos ajustamentos. Por fim, tem-se que os modelos $\mathrm{K} 1, \mathrm{~K} 2$, J2-1 e J2-2 apresentaram-se próximos e com resultados ligeiramente melhores do que os modelos P1 e P2 na primeira distribuição de pontos de apoio. Já na segunda distribuição, a melhora foi mais significativa, atingindo diferenças métricas. Tal fato pode ser visualizado quando se efetua o cálculo dos deslocamentos planimétricos resultantes, conforme apresentado graficamente na Figura 3 a seguir. Em síntese, a utilização de modelos de plataforma polinomiais, com variação linear ou quadrática de $\kappa$, como já esperado, é mais influenciado pela distribuição e número de pontos de apoio na cena, do que os modelos que utilizaram a Equação de Movimento do satélite para modelagem da órbita. 


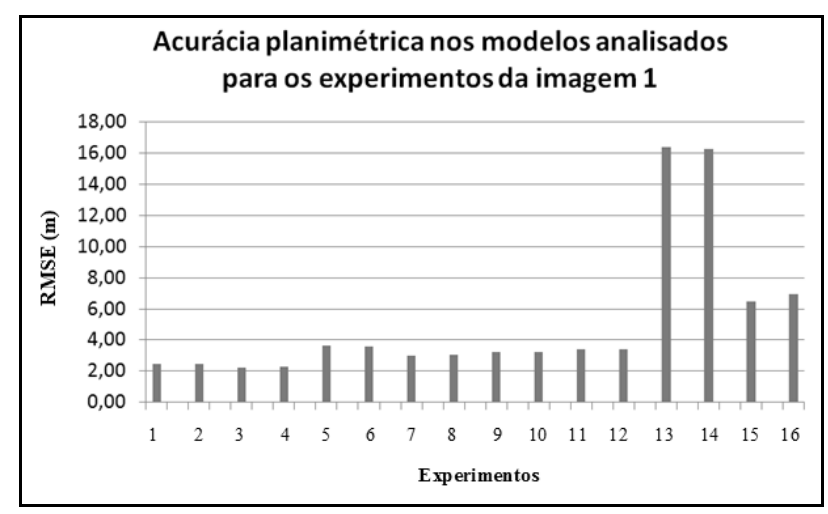

Figura 3: RMSE nos modelos analisados na imagem em visada ré.

Com o objetivo de verificar se houveram tendências nos experimentos, testes de hipótese de tendência, utilizando a distribuição $t$ de student, com nível de confiança de $95 \%$ foram realizados. As hipóteses testadas foram H0: $\mu=0$ e H1: $\mu \neq 0$. O valor de $t$ amostral calculado é dado por:

$$
t=\frac{\mu-\mu_{0}}{s} \sqrt{n}
$$

onde $\mu$ é a média amostral, $\mu_{0}$ é a média populacional esperada (assumida como 0 - sem tendência), $s$ é o desvio padrão amostral e $n$ é o número de pontos de verificação. Na Tabela 6 são apresentados os resultados.

Tabela 6: Testes de tendência para os experimentos da imagem em visada ré.

\begin{tabular}{c|c|c|c|c|c|c|c}
\hline Exp. (MP) & $\begin{array}{c}\mathbf{t} \text { calc./ } \\
\text { Comp. } \mathbf{X}_{\mathbf{L}}\end{array}$ & $\begin{array}{c}\mathbf{t} \text { calc./ } \\
\text { Comp. } \mathbf{Y}_{\mathbf{L}}\end{array}$ & $\mathbf{t}$ (gl;0,975) & Exp. (MP) & $\begin{array}{c}\mathbf{t} \text { calc./ } \\
\text { Comp. } \mathbf{X}_{\mathbf{L}}\end{array}$ & $\begin{array}{c}\mathbf{t} \text { calc./ } \\
\text { Comp. } \mathbf{Y}_{\mathbf{L}}\end{array}$ & $\mathbf{t}$ (gl;0,975) \\
\hline $1(\mathrm{~K} 1)$ & 2,203 & 1,077 & 2,306 & $9(\mathrm{~K} 1)$ & 1,568 & 1,709 & 2,1604 \\
\hline $2(\mathrm{~K} 2)$ & 1,802 & 1,076 & 2,306 & $10(\mathrm{~K} 2)$ & 1,554 & 1,719 & 2,1604 \\
\hline $3(\mathrm{~J} 2-1)$ & 1,908 & 1,256 & 2,306 & $11(\mathrm{~J} 2-1)$ & 1,265 & 1,694 & 2,1604 \\
\hline $4(\mathrm{~J} 2-2)$ & 1,587 & 1,250 & 2,306 & $12(\mathrm{~J} 2-2)$ & 1,251 & 1,694 & 2,1604 \\
\hline $5(\mathrm{ON} 1)$ & 1,703 & 1,312 & 2,306 & $13(\mathrm{ON} 1)$ & 1,097 & 1,098 & 2,1604 \\
\hline $6(\mathrm{ON} 2)$ & 1,675 & 1,394 & 2,306 & $14(\mathrm{ON} 2)$ & 1,115 & 1,116 & 2,1604 \\
\hline $7(\mathrm{P} 1)$ & 2,018 & 1,295 & 2,306 & $15(\mathrm{P} 1)$ & 1,021 & 1,482 & 2,1604 \\
\hline $8(\mathrm{P} 2)$ & 1,990 & 1,209 & 2,306 & $16(\mathrm{P} 2)$ & 0,913 & 1,395 & 2,1604 \\
\hline
\end{tabular}

A partir da análise da Tabela 6 nota-se que nenhum experimento apresentou tendência significativa ao nível de confiança de $95 \%$ para ambas as componentes $\mathrm{X}_{\mathrm{L}}$ e $\mathrm{Y}_{\mathrm{L}}$.

\subsection{Resultados obtidos com a imagem em visada Nadir}

Estes resultados referem-se à aplicação dos modelos apresentados no item 5.1 na orientação da imagem em visada nadir, utilizando as duas configurações de pontos de apoio e verificação, 
sendo denominados experimentos de 17 a 32 respectivamente, conforme indicado na Tabela 7 a seguir.

Tabela 7: Experimentos para a imagem em visada nadir.

\begin{tabular}{cc|cc}
\hline \multicolumn{2}{c|}{ Configuração de pontos de apoio 1 } & \multicolumn{2}{c}{ Configuração de pontos de apoio 2 } \\
\hline Experimento & Modelo & Experimento & Modelo \\
\hline 17 & K1 & 25 & K1 \\
\hline 18 & K2 & 26 & K2 \\
\hline 19 & J2-1 & 27 & J2-1 \\
\hline 20 & J2-2 & 28 & J2-2 \\
\hline 21 & ON1 & 29 & ON1 \\
\hline 22 & ON2 & 30 & ON2 \\
\hline 23 & P1 & 31 & P2 \\
\hline 24 & P2 & 32 &
\end{tabular}

Posteriormente à realização dos ajustamentos, estimou-se os parâmetros de orientação das imagens e suas respectivas precisões. Analogamente aos experimentos realizados na imagem em visada ré, as precisões dos parâmetros em todos os experimentos se mostraram adequadas às ordens de grandeza dos parâmetros. As variações lineares do ângulo $\kappa$ ficaram em média 40"/s para os experimentos de 17 a 20 e de 25 a 28, enquanto que as quadráticas assumiram ordem de grandeza de centésimos de "/s para os experimentos 18, 20, 26 e 28. Para os experimentos de 21 a 24 e de 29 a 32 os padrões de resultado foram idênticos ao grupo de experimentos com a imagem em visada ré, que utilizam os mesmos modelos e configurações de ponto de apoio (experimentos de 5 a 8 e de 13 a 16). Similarmente ao citado nos resultados da imagem em visada ré, a consideração das variações quadráticas de $\kappa$ não foram capazes de alterar significativamente os valores de acurácia, conforme pode ser visto na Tabela 9 dos RMSE dos pontos de verificação.

De forma semelhante à análise dos resultados das orientações na imagem em visada ré, foram também analisados nas orientações da imagem em visada nadir, o teste do Qui-quadrado sobre as variâncias a priori e posteriori, e os RMSE dos vetores dos resíduos. Conforme apresentado na Tabela 8 a seguir, em todos os experimentos houve aceitação da hipótese básica $\left(\hat{\sigma}_{0}^{2}=\sigma_{0}^{2}\right)$ sobre o teste do Qui-quadrado, e, os RMSE das componentes $x_{s}$ e $y_{s}$ dos vetores dos resíduos ficaram próximos ou abaixo do valor de 1 pixel $(0,0071 \mathrm{~mm})$. Com tais resultados para a imagem em visada nadir, pode-se dizer que os modelos matemáticos funcionais utilizados conseguiram modelar adequadamente a realidade física do processo de orientação. Destaca-se aqui que os valores nos vetores dos resíduos apresentaram as mesmas ordens de grandeza das observações, contribuindo para a aceitação das hipóteses básicas, nas duas configurações de pontos de apoio em todos os experimentos. Neste caso, diferentemente dos experimentos 13 e 14, a possível componente sistemática decorrente da aplicação das injunções absolutas $\omega=0^{\circ}$ e $\varphi=0^{\circ}$ não se mostrou significativa. Tal resultado pode estar associado ao fato da imagem em visada nadir ter sido tomada próxima ao nadir. Também semelhantemente ao caso dos experimentos com a imagem em visada ré, em todos os experimentos as médias das componentes dos vetores dos resíduos foram próximas de zero $\left(10^{-6}\right.$ no máximo). 
Tabela 8: Resultados obtidos nos ajustamentos para os experimentos da imagem em visada nadir.

\begin{tabular}{|c|c|c|c|}
\hline $\begin{array}{c}\text { Exp. (MP) com } \\
\text { distribuição de pontos } 1\end{array}$ & Resultados & $\begin{array}{c}\text { Exp. (MP) com } \\
\text { distribuição de pontos } 2\end{array}$ & Resultados \\
\hline 17 (K1) & $\begin{array}{c}\chi_{\text {calculado: }}^{2}: 17,6198 \\
\chi_{\text {teórico }}^{2}(18 ; 0,975): 31,53 \\
\operatorname{RMSE} x_{s}: 0,0074 \mathrm{~mm} \\
\operatorname{RMSE} y_{s}: 0,0036 \mathrm{~mm}\end{array}$ & 25 (K1) & $\begin{array}{c}\chi_{\text {calculado: }}^{2} 9,0213 \\
\chi_{\text {teórico }}^{2}(8 ; 0,975): 17.53 \\
\operatorname{RMSE} x_{s}: 0,0073 \mathrm{~mm} \\
\text { RMSE } y_{s}: 0,0019 \mathrm{~mm}\end{array}$ \\
\hline $18(\mathrm{~K} 2)$ & $\begin{array}{c}\chi_{\text {calculado }}^{2} 16,9440 \\
\chi_{\text {teórico }}^{2}(17 ; 0,975): 30,19 \\
\operatorname{RMSE} x_{s}: 0,0072 \mathrm{~mm} \\
\operatorname{RMSE} y_{s}: 0,0037 \mathrm{~mm}\end{array}$ & 26 (K2) & $\begin{array}{c}\chi_{\text {calculado }}^{2} 9,0050 \\
\chi_{\text {teórico }}^{2}(7 ; 0,975): 16,01 \\
\operatorname{RMSE} x_{s}: 0,0072 \mathrm{~mm} \\
\operatorname{RMSE} y_{s}: 0,0021 \mathrm{~mm}\end{array}$ \\
\hline 19 (J2-1) & $\begin{array}{c}\chi_{\text {calculado }}^{2}: 21,2710 \\
\chi_{\text {teórico }}^{2}(18 ; 0,975): 31,53 \\
\operatorname{RMSE} x_{s}: 0,0083 \mathrm{~mm} \\
\operatorname{RMSE} y_{s}: 0,0038 \mathrm{~mm}\end{array}$ & $27(\mathrm{~J} 2-1)$ & $\begin{array}{c}\chi_{\text {calculado }}^{2}: 10,7892 \\
\chi_{\text {teórico }}^{2}(8 ; 0,975): 17,53 \\
\text { RMSE } x_{s}: 0,0079 \mathrm{~mm} \\
\text { RMSE } y_{s}: 0,0024 \mathrm{~mm}\end{array}$ \\
\hline 20 (J2-2) & $\begin{array}{c}\chi_{\text {calculado }}^{2}: 21,2309 \\
\chi_{\text {teórico }}^{2}(17 ; 0,975): 30,19 \\
\operatorname{RMSE} x_{s}: 0,0082 \mathrm{~mm} \\
\operatorname{RMSE} y_{s}: 0,0039 \mathrm{~mm}\end{array}$ & $28(\mathrm{~J} 2-2)$ & $\begin{array}{c}\chi_{\text {calculado: }}^{2}: 10,7891 \\
\chi_{\text {teórico }}^{2}(7 ; 0,975): 16,01 \\
\operatorname{RMSE} x_{s}: 0,0079 \mathrm{~mm} \\
\operatorname{RMSE} y_{s}: 0,0025 \mathrm{~mm}\end{array}$ \\
\hline 21 (ON1) & $\begin{array}{c}\chi_{\text {calculado }}^{2}: 19,7926 \\
\chi_{\text {teórico }}^{2}(17 ; 0,975): 30,19 \\
\operatorname{RMSE} x_{s}: 0,0074 \mathrm{~mm} \\
\operatorname{RMSE} y_{s}: 0,0046 \mathrm{~mm}\end{array}$ & 29 (ON1) & $\begin{array}{c}\chi_{\text {calculado }}^{2}: 8,1828 \\
\chi_{\text {teórico }}^{2}(7 ; 0,975): 16,01 \\
\operatorname{RMSE} x_{s}: 0,0066 \mathrm{~mm} \\
\operatorname{RMSE} y_{s}: 0,0027 \mathrm{~mm}\end{array}$ \\
\hline 22 (ON2) & $\begin{array}{c}\chi_{\text {calculado }}^{2} 15,8385 \\
\chi_{\text {teórico }}^{2}(16 ; 0,975): 28,85 \\
\operatorname{RMSE} x_{s}: 0,0063 \mathrm{~mm} \\
\operatorname{RMSE} y_{s}: 0,0046 \mathrm{~mm}\end{array}$ & 30 (ON2) & $\begin{array}{c}\chi_{\text {calculado: }}^{2} 2,6666 \\
\chi_{\text {teórico }}^{2}(6 ; 0,975): 14,45 \\
\operatorname{RMSE} x_{s}: 0,0031 \mathrm{~mm} \\
\operatorname{RMSE} y_{s}: 0,0027 \mathrm{~mm}\end{array}$ \\
\hline 23 (P1) & $\begin{array}{c}\chi_{\text {calculado: }}^{2} 10,7595 \\
\chi_{\text {teórico }}^{2}(15 ; 0,975): 27,49 \\
\operatorname{RMSE} x_{s}: 0,0058 \mathrm{~mm} \\
\operatorname{RMSE} y_{s}: 0,0029 \mathrm{~mm}\end{array}$ & 31 (P1) & $\begin{array}{c}\chi_{\text {calculado }}^{2} 6,9935 \\
\chi_{\text {teórico }}^{2}(5 ; 0,975): 12,83 \\
\operatorname{RMSE} x_{s}: 0,0066 \mathrm{~mm} \\
\operatorname{RMSE} y_{s}: 0,0005 \mathrm{~mm}\end{array}$ \\
\hline 24 (P2) & $\begin{array}{c}\chi_{\text {calculado: }}^{2}: 9,7775 \\
\chi_{\text {teórico }}^{2}(14 ; 0,975): 26,12 \\
\operatorname{RMSE~} x_{s}: 0,0054 \mathrm{~mm} \\
\operatorname{RMSE} y_{s}: 0,0029 \mathrm{~mm}\end{array}$ & $32(\mathrm{P} 2)$ & $\begin{array}{c}\chi_{\text {calculado: }}^{2} 0.1802 \\
\chi_{\text {teórico }}^{2}(4 ; 0,975): 11,14 \\
\operatorname{RMSE} x_{s}: 0,0009 \mathrm{~mm} \\
\operatorname{RMSE} y_{s}: 0,0005 \mathrm{~mm}\end{array}$ \\
\hline
\end{tabular}

Com os parâmetros estimados, aplicaram-se os mesmos nos modelos de Colinearidade inversos com a finalidade de investigar a acurácia das orientações. Nos valores de $Z_{i}$ foram utilizados os próprios valores dos pontos de verificação. Todas as coordenadas foram transformadas em coordenadas do Sistema Geodésico Local antes desta análise. A Tabela 9 a seguir apresenta a acurácia nas componentes $\mathrm{X}_{\mathrm{L}}$ e $\mathrm{Y}_{\mathrm{L}}$ em todos os experimentos analisados na imagem em visada nadir. 
Tabela 9: Acurácia nas componentes $X_{L}$ e $Y_{L}$ para os pontos de verificação nos experimentos da imagem em visada nadir.

\begin{tabular}{c|c|c|c|c|c}
\hline $\begin{array}{c}\text { Exp. (Modelo) com } \\
\text { distribuição de pontos 1 }\end{array}$ & \multicolumn{2}{|c|}{ RMSE (m) } & \multicolumn{2}{c}{$\begin{array}{c}\text { Exp. (Modelo) com } \\
\text { distribuição de pontos 2 }\end{array}$} & \multicolumn{2}{c}{ RMSE (m) } \\
\hline $17(\mathrm{~K} 1)$ & 0,937 & 1,915 & $25(\mathrm{~K} 1)$ & 1,712 & 2,767 \\
\hline $18(\mathrm{~K} 2)$ & 1,100 & 1,923 & $26(\mathrm{~K} 2)$ & 1,720 & 2,728 \\
\hline $19(\mathrm{~J} 2-1)$ & 1,062 & 1,865 & $27(\mathrm{~J} 2-1)$ & 1,857 & 2,863 \\
\hline $20(\mathrm{~J} 2-2)$ & 1,125 & 1,865 & $28(\mathrm{~J} 2-2)$ & 1,865 & 2,859 \\
\hline $21(\mathrm{ON} 1)$ & 1,640 & 2,448 & $29(\mathrm{ON} 1)$ & 2,393 & 2,433 \\
\hline $22(\mathrm{ON} 2)$ & 1,614 & 1,630 & $30(\mathrm{ON} 2)$ & 2,191 & 2,620 \\
\hline $23(\mathrm{P} 1)$ & 0,765 & 3,007 & $31(\mathrm{P} 1)$ & 5,156 & 3,872 \\
\hline $24(\mathrm{P} 2)$ & 0,789 & 2,475 & $32(\mathrm{P} 2)$ & 5,122 & 4,667 \\
\hline
\end{tabular}

Efetuando-se o cálculo dos deslocamentos planimétricos resultantes, a comparação gráfica é apresentada na Figura 4 a seguir.

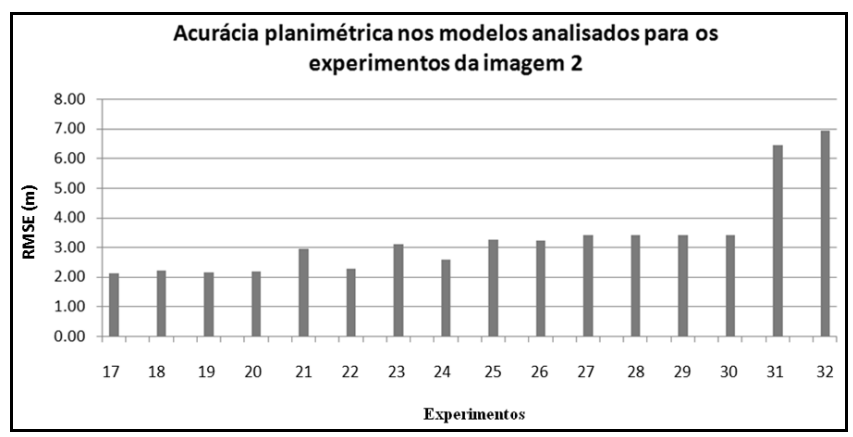

Figura 4: RMSE nos modelos analisados na imagem em visada nadir.

Nota-se a partir da análise da Tabela 9 e da Figura 6 que de forma semelhante ao estudo da imagem em visada ré, a inclusão da variação quadrática do ângulo $\kappa$ não apresentou influencia significativa (diferença máxima na ordem do decímetro) na acurácia das orientações, apresentando-se por vezes pouco melhor ou pior. A inflexibilidade dos modelos polinomiais quanto à quantidade de pontos de apoio e a acurácia posicional, verificada no estudo da imagem em visada ré, ocorreu apenas para os modelos P1 e P2 neste estudo da imagem em visada nadir. Para os demais modelos usando as duas configurações de distribuição de pontos de apoio, os resultados de acurácia planimétrica não se alteraram de forma significativa (menor que 1 pixel). Também de forma contrária ao estudo da imagem em visada ré, os experimentos que utilizaram os modelos ON1 e ON2 apresentaram resultados de acurácia planimétrica mais próximos dos resultados dos outros modelos. Conforme já citado na análise da precisão dos ajustamentos, isso pode ter ocorrido em função da imagem ter sido tomada próxima ao nadir, concordando mais com a ideia das injunções absolutas aplicadas $\omega=0^{\circ}$ e $\varphi=0^{\circ}$. De uma forma geral, para as duas distribuições de pontos de apoio, as acurácias planimétricas obtidas pelos modelos K1, K2, J2-1 e J2-2 apresentaram-se próximas das obtidas pelos demais modelos, com exceção dos modelos $\mathrm{P} 1$ e $\mathrm{P} 2$ nos experimentos 31 e 32. Com relação à adaptação dos modelos K1, K2, J2-1 e J2-2 ao uso de coordenadas referenciadas ao Sistema de Coordenadas Geocêntricas Cartesianas Terrestres mostrou-se adequada, visto os valores de RMSE apresentarem-se em torno de 1 pixel ou pouco mais $(\sim \mathrm{dm})$ que o valor métrico deste. Por fim, observou-se também que a inserção do 
termo que modela a perturbação orbital do satélite proveniente da atração gravitacional terrestre devido a sua elipticidade, não alterou significativamente as acurácias planimétricas, apresentando por vezes resultados ligeiramente melhores ou piores em relação aos modelos que não as consideraram.

Em relação à análise de presença de tendências, realizou-se também testes de hipótese utilizando a distribuição $t$ de student, com as mesmas configurações dos experimentos com a imagem 1 . Os resultados são apresentados na Tabela 10.

Tabela 10: Testes de tendência para os experimentos da imagem em visada nadir.

\begin{tabular}{c|c|c|c|c|c|c|c}
\hline Exp. (MP) & $\begin{array}{c}\mathbf{t} \text { calc./ } \\
\text { Comp. } \\
\mathbf{X}_{\mathbf{L}}\end{array}$ & $\begin{array}{c}\mathbf{t} \text { calc./ } \\
\mathbf{C o m p .} \\
\mathbf{Y}_{\mathbf{L}}\end{array}$ & $\mathbf{t}(\mathbf{g l} ; \mathbf{0 , 9 7 5 )}$ & $\mathbf{E x p . ( M P )}$ & $\begin{array}{c}\mathbf{t} \text { calc./ } \\
\text { Comp. } \mathbf{X}_{\mathbf{L}}\end{array}$ & $\begin{array}{c}\mathbf{t} \text { calc./ } \\
\text { Comp. } \mathbf{Y}_{\mathbf{L}}\end{array}$ & $\mathbf{t}$ (gl;0,975) \\
\hline $17(\mathrm{~K} 1)$ & 1,204 & 1,615 & 2,306 & $25(\mathrm{~K} 1)$ & 1,573 & 1,709 & 2,160 \\
\hline $18(\mathrm{~K} 2)$ & 1,295 & 1,590 & 2,306 & $26(\mathrm{~K} 2)$ & 1,556 & 1,719 & 2,160 \\
\hline $19(\mathrm{~J} 2-1)$ & 1,289 & 1,652 & 2,306 & $27(\mathrm{~J} 2-1)$ & 1,264 & 1,694 & 2,160 \\
\hline $20(\mathrm{~J} 2-2)$ & 1,235 & 1,658 & 2,306 & $28(\mathrm{~J} 2-2)$ & 1,254 & 1,694 & 2,160 \\
\hline $21(\mathrm{ON} 1)$ & 1,803 & 1,676 & 2,306 & $29(\mathrm{ON} 1)$ & 1,694 & 1,269 & 2,160 \\
\hline $22(\mathrm{ON} 2)$ & 1,431 & 0,976 & 2,306 & $30(\mathrm{ON} 2)$ & 1,497 & 1,923 & 2,160 \\
\hline $23(\mathrm{P} 1)$ & 1,142 & 1,337 & 2,306 & $31(\mathrm{P} 1)$ & 1,022 & 1,482 & 2,160 \\
\hline $24(\mathrm{P} 2)$ & 1,175 & 1,280 & 2,306 & $32(\mathrm{P} 2)$ & 0,913 & 1,395 & 2,160 \\
\hline
\end{tabular}

Nota-se a partir da Tabela 10 que, assim como nos experimentos com a imagem 1, nenhum experimento apresentou tendência ao nível de confiança de $95 \%$ para ambas as componentes $\mathrm{X}_{\mathrm{L}}$ e $\mathrm{Y}_{\mathrm{L}}$.

\section{CONCLUSÕES E RECOMENDAÇÕES}

A partir da análise feita sobre os resultados provenientes da utilização da variação linear ou quadrática do ângulo $\kappa$, em ambos os grupos de experimentos, verificou-se que a diferença na acurácia das orientações não se modificou de forma significativa.

Com relação à adaptação dos Modelos de Kepler e órbita perturbada J2 ao uso de coordenadas referenciadas ao Sistema de Coordenadas Geocêntricas Cartesianas Terrestres, para todos os experimentos os resultados se mostraram adequados, tendo em vista que os valores de RMSE alcançaram valores em torno de 1 pixel. Isto possibilita a eliminação da necessidade primária de transformação das coordenadas do espaço objeto para o Sistema de Coordenadas Geocêntrico Cartesiano Inercial.

$\mathrm{Na}$ análise final sobre as acurácias das orientações, para a imagem em visada ré com a primeira distribuição de pontos de apoio, com 14 pontos de apoio e 9 pontos de verificação (Figura 2a), os modelos de Kepler e os modelos considerando a aceleração devido ao geopotencial apresentaram-se próximos, com resultados pouco melhores do que os modelos polinomiais com e sem injunções absolutas em $\omega$ e $\varphi$. Já na segunda distribuição, com 9 pontos de apoio e 14 pontos de verificação (Figura 2b), a melhora foi mais significativa, atingindo diferenças métricas. Para ambas as distribuições de pontos, os experimentos que utilizaram os modelos polinomiais, com injunções absolutas em $\omega$ e $\varphi$, apresentaram resultados menos acurados do que os dos experimentos com os modelos polinomiais sem a aplicação das injunções. A causa de tais 
resultados é a aplicação das injunções absolutas nos ângulos $\omega$ e $\varphi$ ao invés de estimá-los como parâmetros nos ajustamentos.

Ainda com relação às acurácias, no caso da imagem em visada nadir, em ambas as distribuições de pontos de apoio, as acurácias planimétricas obtidas pelos modelos de Kepler, e pelos modelos considerando a aceleração devido ao geopotencial, apresentaram-se próximas das obtidas pelos demais modelos. A exceção ocorreu comparando-se aos resultados do modelo polinomial, sem injunções absolutas em $\omega$ e $\varphi$, na segunda distribuição de pontos de apoio. Ao contrário dos resultados da imagem em visada ré, os experimentos que utilizaram os modelos polinomiais, com injunções absolutas em $\omega$ e $\varphi$, apresentaram resultados de acurácia planimétrica mais próximos dos resultados dos outros modelos, possivelmente pelas injunções absolutas aplicadas $\left(\omega=0^{\circ}\right.$ e $\left.\varphi=0^{\circ}\right)$ representarem mais acuradamente a situação física de obtenção da imagem em visada nadir.

Ao analisar a flexibilidade na quantidade e distribuição de pontos de apoio sobre as imagens, com relação aos resultados de acurácia, houve também divergência. Para a imagem em visada ré observou-se uma menor flexibilidade nos modelos polinomiais, no sentido de que quanto menos pontos, menor a acurácia. Já para a imagem em visada nadir, a quantidade reduzida de pontos proporcionou piores resultados de acurácia somente para os modelos polinomiais sem injunções absolutas em $\omega$ e $\varphi$.

Como apresentado, os resultados das orientações das duas imagens com o modelo considerando a aceleração devido ao geopotencial se mostraram muitos próximos das orientações com o Modelo de Kepler. Ou seja, a inserção no Modelo de Kepler do termo que modela a perturbação orbital do satélite proveniente da atração gravitacional terrestre devido a sua elipticidade, não alterou os resultados de forma significativa em relação ao uso do Movimento Kepleriano no modelo de plataforma. Desta forma, para os casos de orientação exterior de apenas uma imagem, qualquer um dos dois modelos pode ser utilizado. Cabe ressaltar que os resultados para o Modelo de Kepler obtidos nesta pesquisa concordaram em ordem de grandeza com os obtidos por Dowman et al. (2011) e Michalis e Dowman (2010).

Deve-se ainda verificar em trabalhos futuros se há influência ou não da consideração da aceleração proposta pelo potencial gravitacional terrestre devido a sua elipticidade, representado pelo harmônico zonal de grau 2 (J2), nos casos de fototriangulação com imagens na mesma órbita, na mesma data, conforme proposto em Michalis (2005). Isto é, utilizando-se apenas a posição e velocidade da primeira linha da primeira imagem do tripleto PRISM-ALOS e propagando a órbita durante todo o imageamento do tripleto (105 segundos). Recomenda-se ainda a realização de experimentos com menos pontos de apoio e com uma distribuição geométrica ruim, a fim de verificar o potencial dos modelos que utilizam os dados orbitais na estimação dos POEs que descrevem a trajetória da plataforma.

\section{AGRADECIMENTOS}

Os autores agradecem ao Departamento de Cartografia da Faculdade de Ciências e Tecnologia da Universidade Estadual Paulista, campus de Presidente Prudente, por ceder as imagens e pontos de apoio utilizados nesta pesquisa. 


\section{REFERÊNCIAS BIBLIOGRÁFICAS}

Bang, Kiin, and Cho, Woosang "Analysis of EOC Sensor Model". Paper presented at the FIG Working Week, Seoul, Korea, May 6-11, 2001.

Bate, Roger R., Mueller, Donald D., and White, Jerry. Fundamentals of Astrodynamics. Dover, 1971.

Dowman, Ian, and Michalis, Pantelis "Generic rigorous model for along track stereo satellite sensors". Paper presented at the ISPRS workshop on High resolution mapping from Space, Hanover, Niedersachsen, October 4-6, 2003.

Dowman, Ian, Michalis, P., and Li, Zhenhong "Analysis of Urban Landscape Using Multi Sensor Data". Paper presented at the $4^{\text {th }}$ ALOS PI Symposium, Tokyo, Japan, November 15-17, 2010.

Gemael, Camil. Introdução ao ajustamento de observações: aplicações geodésicas. Curitiba: UFPR, 1994.

Gugan, David, and Dowman, Ian. "Topographic mapping from SPOT imagery" Photogrammetric Engineering \& Remote Sensing 54 (1988): 1409-14. Acessed November 11, 2011. doi: 10.1111/j.1477-9730.1987.tb00581.x.

Jacobsen, Karsten "Orientation of high resolution optical space images". Paper presented at the ASPRS 2007 Annual Conference, Tampa, Flórida, May 7-11, 2007.

Japan Aerospace Exploration Agency - JAXA, ALOS User Handbook, 2007. Acessed November 11, 2011. http://www.eorc.jaxa.jp/ALOS/en/doc/alos_userhb_en.pdf.

Jeong, In-seong, and Bethel, James "Trajectory modeling for satellite image triangulation". Paper presented at the XXI ISPRS Congress, Comission I, Beijing, China, July 3-11, 2008.

Kocaman, Sultan, and Armin Gruen "Geometric modeling and validation of ALOS/PRISM imagery and products". Paper presented at the XXI ISPRS Congress, Comission I, Beijing, China, July 3-11, 2008.

Kim, Taejung and Dowman, Ian. "Comparison of two physical sensor models for satellite images: Position-Rotation model and Orbit-Attitude model" The Photogrammetric Record 21 (2006): 110-23. Acessed May 23, 2009. doi: 10.1111/j.1477-9730.2006.00363.x.

Leick, Alfred. GPS Surveying. New Jersey: John Wiley \& Sons, 2004.

Light, Donald L. "Satellite Photogrammetry" In Manual of Photogrammetry, edited by Chester C. Slama, Charles Theurer, and Soren W. Henriksen, 883-977. Virginia: American Society of Photogrammetry, 1980.

Marcato Junior, José and Tommaselli, Antonio Maria Garcia. "Exterior orientation of CBERS2B imagery using multi-feature control and orbital data" ISPRS Journal of Photogrammetry and Remote Sensing 79 (2013): 219-25. Acessed January 27, 2015. doi: 10.1016/j.isprsjprs.2013.02.018.

Marcato Junior et al., "Orientação de imagens CBERS-2B usando o modelo rigoroso de colinearidade com dados orbitais" Boletim de Ciências Geodésicas 17 (2011): 401- 16. Acessed November 11, 2012. doi: 10.1590/S1982-21702011000300005.

Michalis, Pantelis. "Generic rigorous model for along track stereo satellite sensors." $\mathrm{PhD}$ diss., University College London, 2005. 
Michalis, Pantelis, and Dowman, Ian "A rigorous model and DEM generation for SPOT5-HRS". Paper presented at the XX ISPRS Congress, Comission I, Istanbul, Turkey, July 12-23, 2004.

Michalis, Pantelis, and Dowman, Ian. "A Generic Model for Along Track Stereo Sensors Using Rigorous Orbit Mechanics" Photogrammetric Engineering and Remote Sensing 74 (2008a): 30309. Acessed July 13, 2011. doi: 10.14358/PERS.74.3.303.

Michalis, Pantelis, and Dowman, Ian "Exterior orientation improved by the coplanarity equation and DEM generation for Cartosat-1". Paper presented at the XXI ISPRS Congress, Comission I, Beijing, China, July 3-11, 2008 b.

Michalis, Pantelis, and Dowman, Ian "Noise-Reduction and exterior orientation improvement by the coplanarity equation for ALOS-PRISM". Paper presented at the $4^{\text {th }}$ ALOS PI Symposium, Tokyo, Japan, November 15-17, 2010.

Orun, Ahmet B, and Natarajan, Krishnaler . "A Modified Bundle Adjustment Software for SPOT Imagery and Photography: Tradeoff" Photogrammetric Engineering and Remote Sensing 60 (1994): 1431-37.

Poli, Daniela. "A Rigorous Model for Spaceborne Linear Array Sensors" Photogrammetric Engineering and Remote Sensing 73 (2007): 187 - 96. Acessed November 11, 2011. doi: 10.14358/PERS.73.2.187.

Radhadevi, Pullur V, Sasikumar, T.P., and Ramachandran, Rajaraman. "Orbit attitude modelling and derivation of ground co-ordinates from spot stereopairs" Journal of Photogrammetry and Remote Sensing 49 (1994): 22-28. Acessed April 17, 2009. doi: 10.1016/0924-2716(94)90043-4.

Rodrigues, Tiago L, and Ferreira, Luiz Danilo Damasceno "Aplicação do movimento kepleriano na orientação de imagens HRC - CBERS 2B" Boletim de Ciências Geodésicas 19 (2013): 11434. Acessed May 1, 2013. doi: 10.1590/S1982-21702013000100008.

Salamonowick, Paul H. "Satellite orientation and position for geometric correction of scanner imagery" Photogrammetric Engineering and Remote Sensing 52 (1986): 491-99.

Satoru, Watanabe, and Akiriho, Hirao "Development of the Earth Observation Satellite "DAICHI" (ALOS)" NEC Technical Journal 6 (2011): 62-66.

Seeber, Günter. Satellite Geodesy: foundations, methods, and applications. Berlim: Walter de Gruyter, 2003.

Toledo et al., "Fototriangulação em bloco de imagens ALOS/PRISM para mapeamento na escala 1:25000 da região de Presidente Prudente - SP" Trabalho de conclusão de curso de graduação, Universidade Estadual Paulista, 2010.

Torgue, Wolfgang. Geodesy. Berlin; New York: Walter de Gruyter, 2001.

Toutin, Thierry "Geometric processing of Remote Sensing images: models, algorithms and methods" International Journal of Remote Sensing 25 (2004): 1893-924. Acessed April 13, 2009. doi: 10.1080/0143116031000101611.

Weser et al., "Development and testing of a generic sensor model for pushbroom satellite imagery" The Photogrammetric Record 23 (2008): 255-74. Acessed November 11, 2011. doi: 10.1111/j.1477-9730.2008.00489.x.

Recebido em Novembro de 2014. Aceito em Março de 2015. 